\title{
Far-field flat lens based on multilayered metal- dielectric structure
}

\author{
Vladimir Belyi $^{1}$, Mohammed Binhussain ${ }^{2}$, Nikolai Khilo ${ }^{1}$, Nikolai Kazak ${ }^{1}$ \\ ${ }^{1}$ Insitute of Physics of NAS Belarus, Minsk, Belarus \\ ${ }^{2}$ The National Center for Building System, KACST, Riyadh, Saudi Arabia \\ *corresponding author, E-mail: v.belyi@dragon.bas-net.by
}

\begin{abstract}
The detailed investigation has been made of the lens effect in plane multilayered metal-dielectric structures $\left(\mathrm{Ag}-\mathrm{TiO}_{2}\right)$. The optical scheme of the lens has been studied with the radiation focusing in free space. The transfer function is calculated, where the phase profile determines definitely the possibility of focusing. The condition for far- field image formation is found using a flat lens. This condition is used for a numerical simulation of several lens designs with a various number of metal layers. It is found that considered flat lenses have close to limiting angular aperture and therefore the subwavelength resolution. It is established that at increase of a number of metal layers the object and image distances grow.
\end{abstract}

\section{Introduction}

One of the most important achievements in optics of metamaterials is to establish the possibility of light focusing by plane-parallel multi-layered metal-dielectric structures [1-3]. Lens effect in such structures is interesting for the optical microscopy, fluorescence imaging, lithography and so on. For a successful application of flat lens further it is necessary to explain not only the physical fundamentals of their functioning, but also to calculate and analyze their characteristics. Flat lenses in this respect differ advantageously from other nanostrucutres such as structures with nanocylinders, because lenses allow accurate calculations. In the study of flat lenses there is no necessity to use approximation of effective permeability or to introduce anisotropy. At the same time the comparison of the results of exact calculation with approximate ones allows one to understand deeper the physical meaning of approximations and to determine the limits of their applicability. Of course, this is important for further use of the approximate methods at calculations of more complex nanostructures.

\section{Optical scheme of flat lens}

The optical scheme illustrated in Fig. 1 will be investigated. Here a layered metal-dielectric (MD) system is illuminated by a divergent light beam from the source located at the distance $z_{1}$ from the layered structure. The layered structure consists of several layers of metal and dielectric (in the studied case of $\mathrm{Ag}$ and $\mathrm{TiO}_{2}$ ). Homogeneous media on both sides of lens in the general case are different (Fig.1b).
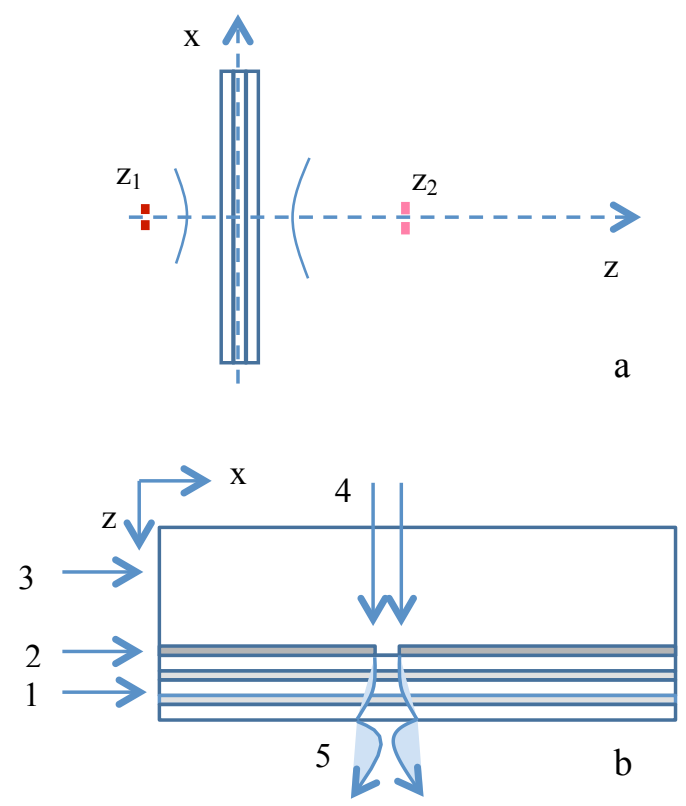

Figure 1. The principal scheme of far-field flat lens (a). The scheme of the lens calculated in the paper (b).

Here: 1 - layered metal-dielectric system; 2 - nontransparent coating with one or several apertures; 3 substrate; 4 - incident light beam; 5 - beam at the lens output.

Under certain conditions, which will be specified hereinafter, MD- system acts as a lens that means that at some distance $z_{2}$ source image is formed. The optical scheme of the lens, as it is presented in Fig.1b, makes focusing into free space. That is why the incident field propagates from the side of substrate. To investigated the focusing it is enough to use the scheme with one incident beam, but at calculation of spatial resolution also the scheme can be used with two close apertures, i.e. with illumination of lens by two parallel beams.

Far-field imaging by the lens is realized in a pure form if the source does not create an evanescent field or if distance $\mathrm{z}_{1}$ is rather large and the evanescent field (at the lens input) disappears. In this case in the image plane the field will not be an evanescent one. The same regime of imaging takes place also when distance $z_{1}$ is short, but distance $z_{2}$ is large. Fig. 1a illustrates also equiphase surfaces within the region of the source and the image. The presence of concave surface (in the image plane) within the region of 
the image there is a necessary condition of functioning of lens in the regime of far-field imaging.

Finally, it should be pointed out that unlike conventional optical lenses the most important parameter of flat lenses is their energy efficiency. This is due to the presence of lightabsorbing metal layers, number of which can be more than one.

\section{Theory}

To obtain the quantitative characteristics of flat lens the accurate method based on calculation the transfer matrix (TM) for TH- polarized field will be used. Applied to the vector field, the TM for $\mathrm{x}$ - component of electric field and $\mathrm{y}$ component of magnetic field will be calculated. It will allow us to calculate the $\mathrm{z}$ - component of Pointing vector and at last the power efficiency of flat lens.

The relation between $\mathrm{x}$-components of input (in) and output (out) electric fields for the flat lens it is possible to represent as

$$
E_{\text {out }, x}\left(n_{x}\right)=n_{\text {out }, z}\left(n_{x}\right) E_{\mathrm{in}, x}\left(n_{x}\right) / M_{1,1}^{-1}\left(n_{x}\right) .
$$

Here the particular case is studied of two-dimensional space of wave numbers $\left(k_{x}, k_{z}\right)$ and hence the normalized transverse wave number $n_{x}=k_{x} / k_{0}$ is introduced, where $k_{0}=2 \pi / \lambda$ and $k_{z}=k_{0} \sqrt{\varepsilon-n_{x}^{2}} . M_{1,1}$ is the component of TM of multilayer.

As it follows from the optical scheme the lens, their TM is a product of three multipliers $M=M_{\text {freel }} M_{m l} M_{\text {free2 }}$, where indices (free) and $(\mathrm{ml})$ relate to free space and multilayer.

The ability of image formation by the optical scheme under study is mainly determined by phase function in the relation as follows from Eq. (1)

$$
E_{\text {out }, x}\left(n_{x}\right)=t\left(n_{x}\right) \exp \left(i \varphi\left(n_{x}\right)\right) E_{\text {in }, x}\left(n_{x}\right),
$$

If function $\varphi\left(n_{x}\right)$ depends weakly on the transverse wave number and function $t\left(n_{x}\right)$ is rather smooth in comparison with the source field, then the scheme in Fig. 1 is considered to be as a flat lens. Let function $\varphi\left(n_{x}\right)$ be in a form of sum

$$
\varphi\left(n_{x}\right)=\varphi_{\text {free }}\left(n_{x}\right)+\varphi_{m l}\left(n_{x}\right)
$$

where

$\varphi_{\text {free }}\left(n_{x}, \mathrm{z}_{1}, \mathrm{z}_{2}\right)=k_{0} \sqrt{\varepsilon_{\text {in }}-n_{x}^{2}} z_{1}+k_{0} \sqrt{\varepsilon_{\text {out }}-n_{x}^{2}} z_{2}$

and $\varphi_{m l}\left(n_{x}\right)$ is the phase function of multilayer.

In the Eq (4) the phase function of free space is positive. But it does not follow from it that for compensation of phase incursion phase function of multilayer should be negative. It is only important that function $\varphi_{m l}\left(n_{x}\right)$ increases while $n_{x}$ becomes larger.

Then the condition of realization of the flat lens has the form

$$
\varphi\left(n_{x}\right) \approx \mathrm{const}
$$

The deviation of function $\varphi\left(n_{x}\right)$ from the constant value will be considered as a source of aberrations.

\section{Calculation and analysis of specific systems}

The simplest experimentally realized system is the layer of metal on the dielectric substrate. Let's study the system $\mathrm{SiO}_{2}$ (substrate) $-\mathrm{Ag}(20 \mathrm{~nm})-\mathrm{TiO}_{2}(20 \mathrm{~nm})$.
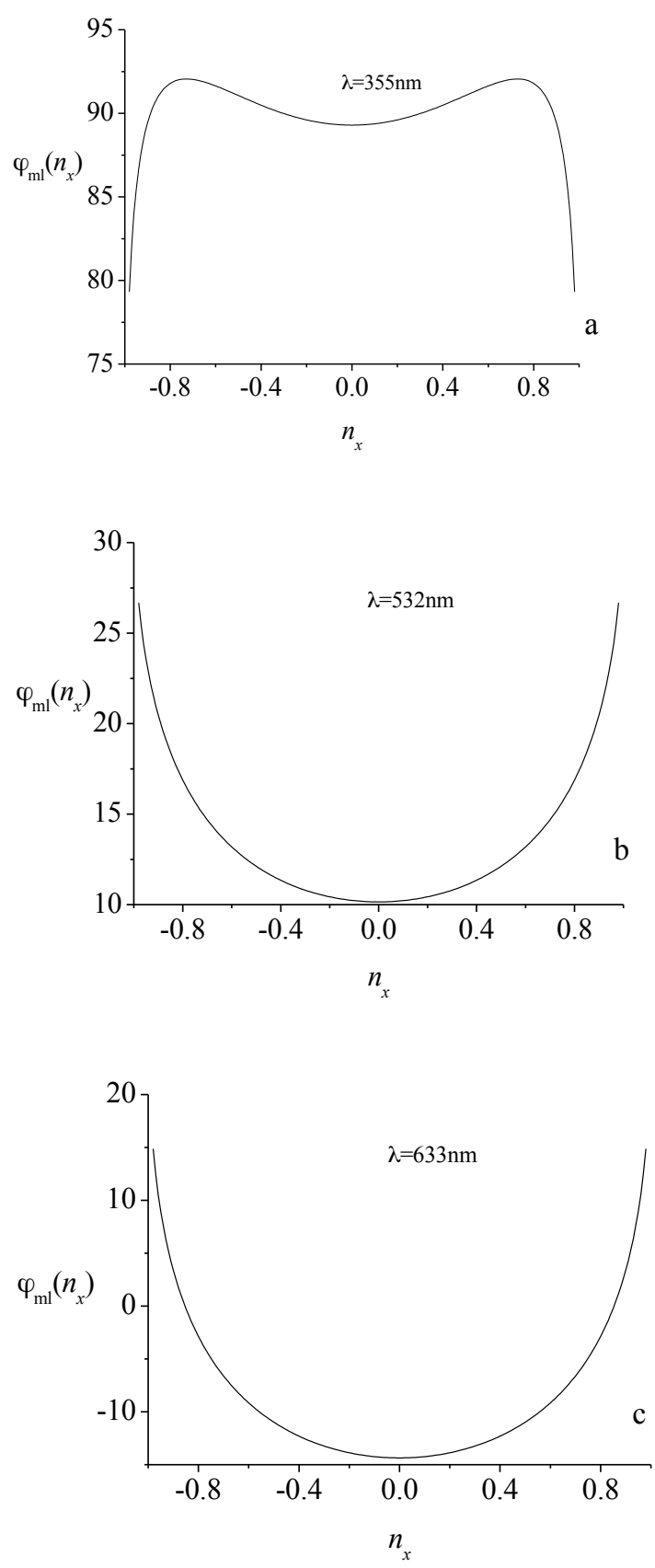


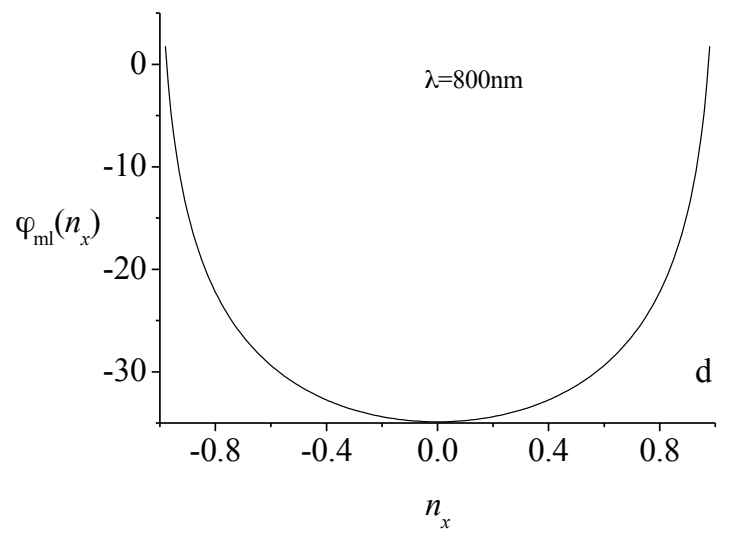

Figure 2. Phase functions of multilayer at wavelength $\lambda=$ $355 \mathrm{~nm}(\mathrm{a}) ; 532 \mathrm{~nm}(\mathrm{~b}) ; 633 \mathrm{~nm}(\mathrm{c}) ; 800 \mathrm{~nm}(\mathrm{~d})$; Hereinafter the phase is measured in degrees.

As is seen from Fig.2, the phase function of this multilayer is concave almost in all considered cases. Hence the average phase shift decreases with the increase of the wavelength and becomes negative in near IR-region. But in this region the lens is the least energy-efficient.

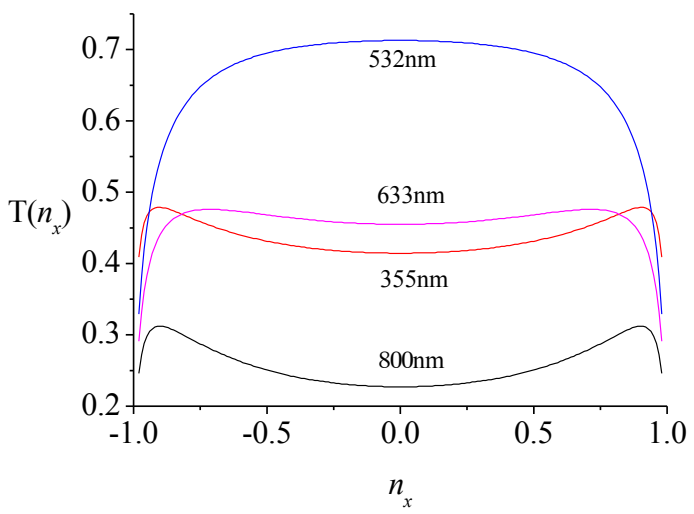

Figure 3. Angular dependence of the transmission coefficient for various wavelengths.

The above-mentioned least energy-efficiency is seen from Fig. 3, which illustrates that the transmission of the lens depends non-monotonically on the wavelength and is rather large for the wavelengths under study, except for $\lambda=$ $800 \mathrm{~nm}$

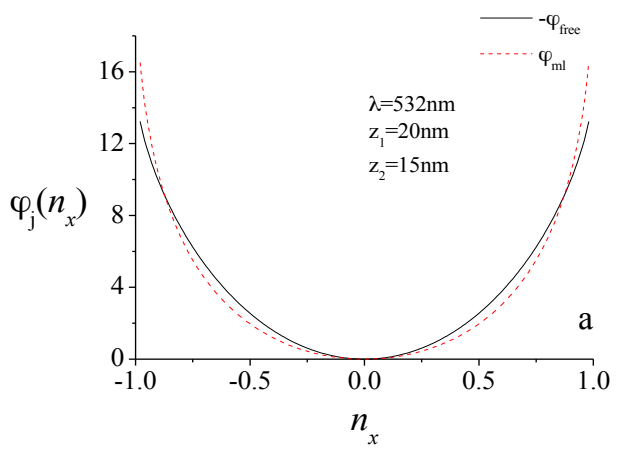

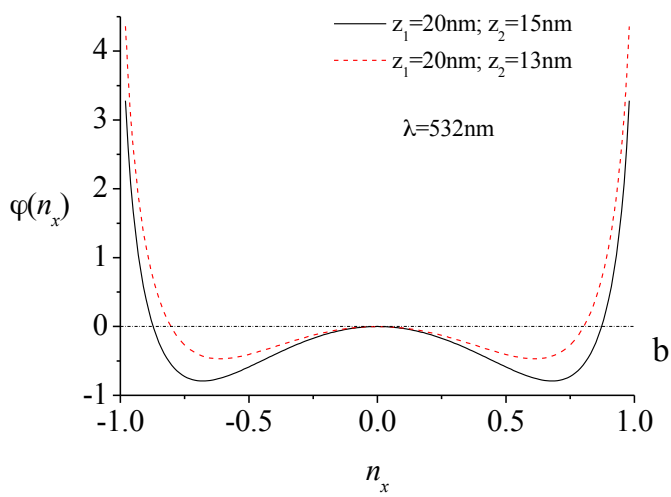

Fig. 4 Phase functions $-\varphi_{\text {free }}\left(n_{x}, 20,15\right)$ (solid) and $\varphi_{m l}\left(n_{x}\right)$ (dash) (a) and total phase function $\varphi\left(n_{x}\right)$ (b).

Fig. 4 illustrates the degree of fulfillment of the approximation (5) for the studied scheme. The function $\varphi_{\text {free }}\left(n_{x}, \mathrm{z}_{1}, \mathrm{z}_{2}\right)$ can vary due to the change of distances $\mathrm{z}_{1}$ and $z_{2}$, selecting them in such a way that difference of curves in Fig. 4a was minimal. Hence the minimal values of both functions have been chosen equal to zero by subtracting the phase shifts at $n_{x}=0$. Fig. $4 \mathrm{~b}$ shows the phase function $\varphi\left(n_{x}\right)$ (Eq. 3). As is seen in Fig. 4, the difference of phase shift is located within \pm 1 degrees except for the extreme regions corresponding to close to grazing incidence. It means the considered lens has close to limiting angular aperture and hence the subwavelength resolution.

It should be also noted that for a given $z_{1}$ and slightly different values $z_{2}$ the phase distortion produced by the lens has a different character. According to Fig. $4 \mathrm{~b}$ at change of $\mathrm{Z}_{2}$ from $15 \mathrm{~nm}$ up to $13 \mathrm{~nm}$ the distortions in the area of low spatial frequencies (SF) decrease and in the area of high SF increase. For full estimation of the quality of focusing in such cases it is necessary to take into account dependence of the lens transmission coefficient on the SF. In the case under the study, as follows from Fig. 3, lens transmission for $\lambda=$ $532 \mathrm{~nm}$ in the area of high SF is sharply decreasing. That is why the phase distortion in this area can be neglected in first approximation. Fig. 3 shows also that the degree of the fall of transmission function at high SF can depend essentially on the wavelength. So for description of the flat lens it is reasonable to introduce also the parameter of angular aperture $n_{x, \max }$. For example, for the case considered above $n_{\mathrm{x}, \max }=0.975$ in accordance with criterion of fall of transmission function in two times.

At the given parameter $n_{x, \max }$ the value has been investigated of the average phase error, which has been calculated using the formula

$\operatorname{Err}\left(\lambda, \mathrm{z}_{1}, \mathrm{z}_{2}\right)=\int_{0}^{n_{x, \max }}\left|\varphi\left(\lambda, n_{x}, \mathrm{z}_{1}, \mathrm{z}_{2}\right)\right| \mathrm{d} n_{x}$

At a given value $z_{1}$ the minimum of the integral depending on $z_{2}$ has been found. It is revealed that the minimal value of 
integral (6) exists and depends on the magnitude $z_{1}$. Hence within the range $z_{1}$ from $14 \mathrm{~nm}$ up to $22 \mathrm{~nm}$ the distances $z_{1}$ and $\mathrm{Z}_{2}$ satisfy approximately the simple lens formula $z_{1}^{-1}+z_{2}^{-1}=\mathrm{f}^{-1}$ with the value $\mathrm{f} \approx 7.8 \mathrm{~nm}$.

The obtained result, however, has some limitations of the physical character. It is meant that within the range of values $\mathrm{z}_{1}$ and $\mathrm{z}_{2}$, pointed above, the far field will not exist in a pure form, because its overlap is possible with the evanescent field.

That is why the task is acute to search the possibilities of increasing the focal distance of such a type of lenses. Apparently, the only solution here is to move to the systems with more large number of layers. Consider for example, the structure $\mathrm{Ag}-\mathrm{TiO}_{2}-\mathrm{Ag}-\mathrm{TiO}_{2}-\mathrm{Ag}-\mathrm{TiO}_{2}$ on $\mathrm{SiO} 2$ substrate. The individual layer thicknesses are 33, 28, 30, 28, 33 and $10 \mathrm{~nm}$ respectively. Such a structure has been used in paper [5] as unite cell of more complex flat lens. Here layer $\mathrm{TiO}_{2}(10 \mathrm{~nm})$ is additionally introduced for modeling the protective coating of metal. Unlike [5] here the focusing not in substrate but in air is studied.

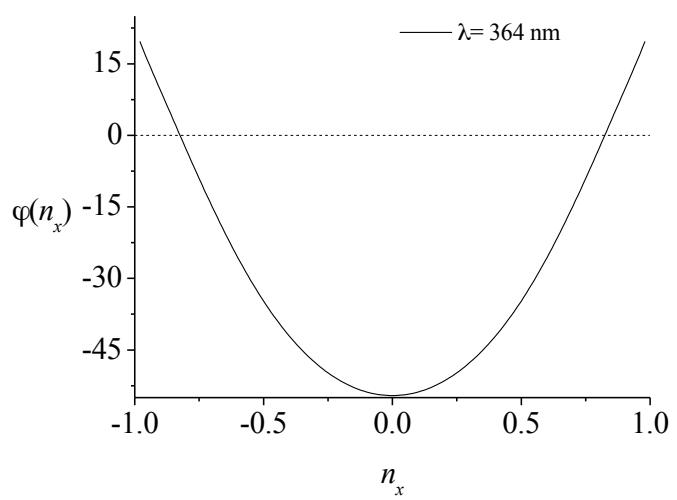

Figure 5. Phase function of the MD system containing three layers of metal.

Phase characteristic of lens is shown in Fig.5. As is seen, the phase shift of light when passing through the lens is negative, except for small edge areas. But these areas are not essential that follows from Fig. 6 where the angular dependence of transmission of this lens is shown. As was expected, its energy efficiency reduces essentially. Parameter $n_{\mathrm{x}, \max }$ here is approximately equal to 0.75 .

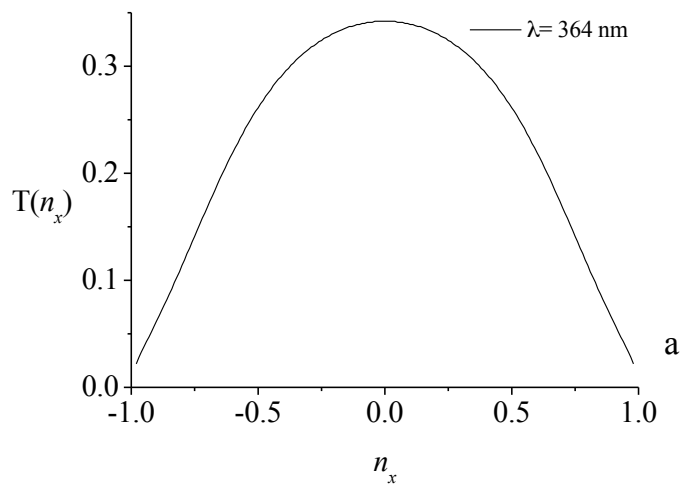

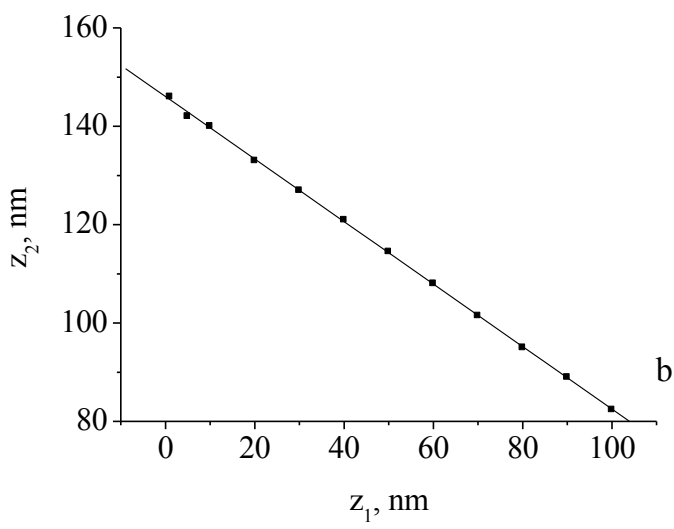

Figure 6. Transmission function of flat lens (a). Dependence $\mathrm{z}_{2}\left(\mathrm{z}_{1}\right)$ providing the minimum of phase distortion. Wavelength $\lambda$ is equal to $364 \mathrm{~nm}$.

Further the calculation has been made of lengths $z_{1}$ and $z_{2}$, at which the phase distortion of lens is minimal. Fig. $6 b$ illustrates dependence $z_{2}\left(z_{1}\right)$ and its approximation by straight line. The equation of straight line has the form

$z_{2}=\operatorname{tg}(\theta)\left(\mathrm{z}_{0}-\mathrm{z}_{1}\right)$

Here $\theta$ is the acute angle of the line with axis $z_{1}$. In the case under study $\theta$ is equal to 32.4 degrees, and $z_{0}=230 \mathrm{~nm}$. The relationship of these parameters with the characteristics of multilayer is not still clear.

It should be also noted that the thin- lens- equation obtained before is not fulfilled here.

The plots in Fig. $6 \mathrm{~b}$ shows that object distance $\mathrm{z}_{1}$ and image distance $z_{2}$, as it is supposed, are essentially larger that for MD- lens with single metallic layer.

\section{Conclusions}

In the paper the description is made of flat lenses consisting of layered MD- structures. The description is based on the application of transfer matrix, which is calculated numerically. Using the established components of transfer matrix further the transfer function is calculated of the layered structure and its phase characteristics. Also transmission function of lens is established determining its energy efficiency. At the next stage the phase distortion is calculated of flat far-field lens in comparison with ideally imaging lens. From the condition of minimum of phase distortions the function is determined of relation between object $z_{1}$ and image $z_{2}$ distances. The particular cases have been studied of layered structures with $\mathrm{Ag}$ and $\mathrm{TiO}_{2}$ as the constituent materials on substrate of $\mathrm{SiO}_{2}$, which differs by the number of metal and dielectric layers. It is shown that for the lens with a single Ag- layer the values $z_{1}$ and $z_{2}$ are small and located in the region of $10 \div 20 \mathrm{~nm}$. For lens with three layers of $\mathrm{Ag}$ the distances increase up to values of order of $100 \mathrm{~nm}$. This trend is preserved further. Thus, the doubling of the second structures described above, the optimal values of $z_{1}$ and $z_{2}$ can be equal to $150 \mathrm{~nm}$ and $223 \mathrm{~nm}$, respectively. However, the transmittance of this lens 
is small and is at the level of several percent. Moreover, with the increase of the number of layers the phase characteristic of lens is deformed, i.e. phase distortions increase. The decrease of distortions is possible at reduction of the angular divergence of the input field that leads to a reduction in spatial resolution.

In a whole, as the calculations and analysis showed, flat lenses on the basis of layered MD structures are characterized by rather smooth transfer functions, parameters of which can be predictably changed due to the choice of geometric parameters of the layered structure.

\section{Acknowledgements}

The authors would like to thank King Abdul-Aziz City for Science and Technology (KACST) for Research Grant No. 814-33.

\section{References}

[1] J. B. Pendry. Negative refraction makes a perfect lens, Phys. Rev. Lett. 85: 3966-3969, 2000.

[2] S. Durant, Z. Liu, J. M. Steele, and X. Zhang, Theory of the transmission properties of an optical far-field superlens for imaging beyond the diffraction limit, $J$. Opt. Soc. Am. B 23: 2383-2392, 2006.

[3] D. Lu, Z. Liu. Hyperlenses and metalenses for farfield super-resolution imaging, Nature Communications 3:1205, 1-9, 2012.

[4] Yeh, P. Optical Waves in Layered Media, 118-143, Wiley, 1988.

[5] T. Xu, A. Agrawal, M. Abashin, K. J. Chau, H. J. Lezec. All-angle negative refraction and active flat lensing of ultraviolet light, Nature 497: 470-474, 2013. 